BULLETIN (New Series) OF THE

AMERICAN MATHEMATICAL SOCIETY

Volume 31, Number 1, July 1994

\title{
SELBERG'S CONJECTURES AND ARTIN $L$-FUNCTIONS
}

\author{
M. RAM MURTY
}

\section{INTRODUCTION}

In its comprehensive form, an identity between an automorphic $L$-function and a "motivic" $L$-function is called a reciprocity law. The celebrated Artin reciprocity law is perhaps the fundamental example. The conjecture of ShimuraTaniyama that every elliptic curve over $Q$ is "modular" is certainly the most intriguing reciprocity conjecture of our time. The "Himalayan peaks" that hold the secrets of these nonabelian reciprocity laws challenge humanity, and, with the visionary Langlands program, we have mapped out before us one means of ascent to those lofty peaks. The recent work of Wiles suggests that an important case (the semistable case) of the Shimura-Taniyama conjecture is on the horizon and perhaps this is another means of ascent. In either case, a long journey is predicted. To paraphrase the cartographer, it is not a journey for the fainthearted. Indeed, there is a forest to traverse, "whose trees will not fall with a few timid blows. We have to take up the double-bitted axe and the cross-cut saw and hope that our muscles are equal to them."

At the 1989 Amalfi meeting, Selberg [S] announced a series of conjectures which looks like another approach to the summit. Alas, neither path seems the easier climb. Selberg's conjectures concern Dirichlet series, which admit analytic continuations, Euler products, and functional equations.

The Riemann zeta function is the simplest example of a function in the family $\mathscr{S}$ of functions $F(s)$ of a complex variable $s$ satisfying the following properties:

(i) (Dirichlet series) For $\operatorname{Re}(s)>1, F(s)=\sum_{n=1}^{\infty} a_{n} / n^{s}$, where $a_{1}=1$, and we will write $a_{n}(F)=a_{n}$ for the coefficients of the Dirichlet series.

(ii) (Analytic continuation) $F(s)$ extends to a meromorphic function so that for some integer $m \geq 0,(s-1)^{m} F(s)$ is an entire function of finite order.

(iii) (Functional equation) There are numbers $Q>0, \alpha_{i}>0, \operatorname{Re}\left(r_{i}\right) \geq 0$, so that

$$
\Phi(s)=Q^{s} \prod_{i=1}^{d} \Gamma\left(\alpha_{i} s+r_{i}\right) F(s)
$$

satisfies $\Phi(s)=w \overline{\Phi(1-\bar{s})}$ for some complex number $w$ with $|w|=1$.

(iv) (Euler product) $F(s)=\prod_{p} F_{p}(s)$ where $F_{p}(s)=\exp \left(\sum_{k=1}^{\infty} b_{p^{k}} / p^{k s}\right)$ where $b_{p^{k}}=O\left(p^{k \theta}\right)$ for some $\theta<1 / 2$, where $p$ denotes a prime number (here and throughout this paper).

(v) (Ramanujan hypothesis) $a_{n}=O\left(n^{\epsilon}\right)$ for any fixed $\varepsilon>0$.

Received by the editors September 4, 1992, and, in revised form, September 28, 1993.

1991 Mathematics Subject Classification. Primary 11M41, 11 R42.

Research partially supported by an NSERC grant. 
Note that the family $\mathscr{S}$ is multiplicatively closed and so is a multiplicative monoid.

All known examples of elements in $\mathscr{S}$ are automorphic $L$-functions. In all of these cases, $F_{p}(s)$ is an inverse of a polynomial in $p^{-s}$ of bounded degree.

Selberg [S] introduced this family to study the value distribution of finite linear combinations of Dirichlet series with Euler products and functional equations. For this purpose, he introduced the important concept of a primitive function and made significant conjectures about them.

A function $F \in \mathscr{S}$ is called primitive if the equation $F=F_{1} F_{2}$ with $F_{1}, F_{2} \in \mathscr{S}$ implies $F=F_{1}$ or $F=F_{2}$. As we shall see below, one of the most serious consequences of the Selberg conjectures is that $\mathscr{S}$ has unique factorization into primitive elements. It is not difficult to show that every element of $\mathscr{S}$ can be factored into primitive elements. This is a consequence of an old theorem of Bochner [B], though Selberg [S] and more recently Conrey and Ghosh [CG] seem to have found it independently.

Selberg conjectures:

Conjecture A. For all $F \in \mathscr{S}$, there exists a positive integer $n_{F}$ such that

$$
\sum_{p \leq x} \frac{\left|a_{p}(F)\right|^{2}}{p}=n_{F} \log \log x+O(1) .
$$

In Proposition 2.5, we shall describe $n_{F}$ more explicitly.

Conjecture B. (i) For any primitive function $F, n_{F}=1$ so that

$$
\sum_{p \leq x} \frac{\left|a_{p}(F)\right|^{2}}{p}=\log \log x+O(1) .
$$

(ii) For two distinct primitive functions $F$ and $F^{\prime}$,

$$
\sum_{p \leq x} \frac{a_{p}(F) \overline{a_{p}\left(F^{\prime}\right)}}{p}=O(1) .
$$

Thus, in some sense, the primitive functions form an orthonormal system.

In his paper [S], Selberg investigates the consequences of his conjectures to the value distribution of $\log F(\sigma+i t)$ for $\sigma=1 / 2$ or $\sigma$ very near to $1 / 2$. Selberg also conjectures the analogue of the Riemann hypothesis for the functions $F \in$ $\mathscr{S}$.

It is not difficult to see that Conjecture B implies Conjecture A. By Proposition 2.4, Conjecture B also implies that the factorization into primitives in $\mathscr{S}$ is unique. It seems central, therefore, to classify the primitive functions.

To this end, it is natural to define the dimension of $F$ as

$$
\operatorname{dim} F=2 \alpha_{F}
$$

where

$$
\alpha_{F}=\sum_{i=1}^{d} \alpha_{i}
$$

By Proposition 2.2, this concept is well defined. Selberg conjectures that the dimension of $F$ is always a nonnegative integer. This question was previously raised by Vignéras [V]. 
Bochner's work can be used to classify primitive functions of dimension one in the case $\alpha_{1}=1 / 2$. They are (after a suitable translation) the classical zeta function of Riemann and the classical $L$-functions of Dirichlet. If the $\alpha_{i}$ are rational numbers, then this is also a complete list of (primitive) functions of dimension one (see [M2]).

The main goal of this paper is to show that Conjecture B implies Artin's conjecture concerning the holomorphy of nonabelian $L$-series attached to irreducible Galois representations. More precisely, let $k$ be an algebraic number field and $K / k$ a finite Galois extension with group $G$. Let $\rho$ be an irreducible representation on the $n$-dimensional complex vector space $V$. For each prime ideal $\mathfrak{p}$ of $k$, let $V_{\mathfrak{p}}$ be the subspace of $V$ fixed by the inertia group $I_{\mathfrak{p}}$ of $\mathfrak{p}$. Set

$$
L_{\mathfrak{p}}(s, \rho)=\operatorname{det}\left(1-\rho\left(\sigma_{\mathfrak{p}}\right) \mathbf{N p}^{-s} \mid V_{\mathfrak{p}}\right)^{-1}
$$

where $\sigma_{\mathfrak{p}}$ is the Frobenius automorphism of the prime ideal $\mathfrak{p}$ of $k$ and $\mathbf{N}$ is the absolute norm from $k$ to $Q$. Define

$$
L(s, \rho ; K / k)=\prod_{\mathfrak{p}} L_{\mathfrak{p}}(s, \rho) .
$$

(Sometimes we write $L(s, \rho)$ if the field extension is clear. Since $L(s, \rho)$ depends only on the character $\chi$ of $\rho$, we will also sometimes write $L(s, \chi)$ or $L(s, \chi, K / k)$ for $L(s, \rho, K / k)$.) Clearly, the Artin $L$-function $L(s, \rho)$ is a product of $L$-functions attached to irreducible constituents of $\rho$. We have:

Artin's conjecture. If $\rho$ is irreducible $\neq 1$, then $L(s, \rho, K / k)$ extends to an entire function of $s$.

This embodies one of the central problems of number theory. Langlands [L1] has enunciated an even stronger conjecture: if $n=\operatorname{dim} \rho$, then there is a cuspidal automorphic representation $\pi$ of $G L_{n}\left(A_{k}\right)$, where $A_{k}$ is the adele ring of $k$, so that

$$
L(s, \rho, K / k)=L(s, \pi) .
$$

This is called the Langlands reciprocity conjecture. In case $\rho$ is one-dimensional, the conjecture reduces to the celebrated Artin reciprocity law.

Using Artin's reciprocity law, we will show that Conjecture B implies even the Langlands reciprocity conjecture if $K / Q$ is solvable. The constraint of solvability arises from the work of Arthur and Clozel [AC], who showed that the maps of base change and automorphic inductions for automorphic representations exist if $K / k$ is cyclic of prime degree. The maps are, however, conjectured to exist in general. If such is the case, then the Langlands reciprocity conjecture is true in its full generality (see [M1]). In fact, it will be apparent from Theorem 4.3 and Remark 4.4 that if the Dedekind zeta function of $K$ is the $L$-function of an automorphic representation over $Q$, then Conjecture B implies the Langlands reciprocity conjecture.

The concept of a primitive function is an important one. The Selberg conjectures imply that $L$-functions attached to irreducible cuspidal automorphic representations over $Q$ are primitive. It would be extremely interesting to determine whether there are elements of $\mathscr{S}$ which do not arise from automorphic representations. 


\section{Preliminaries}

We record in this section the results of Bochner [B], Selberg [S], and ConreyGhosh [CG].

Proposition 2.1 (Bochner [B]). If $F \in \mathscr{S}$ and $\alpha_{F}>0$, then $\alpha_{F} \geq 1 / 2$.

Remark. In their paper, Conrey and Ghosh [CG] give a simple proof of this and also treat the case $\alpha_{F}=0$. They prove that the constraint $b_{n}=O\left(n^{\theta}\right)$ for some $\theta<1 / 2$ implies there is no element in $\mathscr{S}$ with $\alpha_{F}=0$ except the constant function 1 .

Proposition 2.2 (Selberg [S]). Let $N_{F}(T)$ be the number of zeros $\rho=\beta+i \gamma$ of $F(s)$ satisfying $0<\gamma \leq T$. Then

$$
N_{F}(T)=\frac{\alpha_{F}}{\pi} T(\log T+c)+S_{F}(T)+O(1),
$$

where $c$ is a constant and $S_{F}(T)=O(\log T)$.

If $F=F_{1} F_{2}$, then clearly $N_{F}(T)=N_{F_{1}}(T)+N_{F_{2}}(T)$ so that $\alpha_{F}=\alpha_{F_{1}}+\alpha_{F_{2}}$. Thus, if $F$ is such that $\alpha_{F}<1$, then $F$ is necessarily primitive. The following is now immediate.

Proposition 2.3 [CG]. Every $F \in \mathscr{S}$ has a factorization into primitive functions. Proof. If $F$ is not primitive, then $F=F_{1} F_{2}$, and, by the above, $\alpha_{F}=\alpha_{F_{1}}+\alpha_{F_{2}}$. By Proposition 2.1, each of $\alpha_{F_{1}}, \alpha_{F_{2}}$ is strictly less than $\alpha_{F}$. Continuing this process, we find that the process terminates because Proposition 2.1 implies the number of factors is $\leq 2 \alpha_{F}$. This completes the proof.

We see immediately that the Riemann zeta function and the classical Dirichlet functions $L(s, \chi)$ with $\chi$ a primitive character are primitive in the sense of Selberg. Indeed, the $\Gamma$-factor appearing in the functional equation is $\Gamma(s / 2)$ or $\Gamma((s+1) / 2)$, and the result is now clear from Proposition 2.1.

Conjecture B forces the factorization in Proposition 2.3 to be unique. Indeed, suppose that $F$ had two factorizations into primitive functions:

$$
F=F_{1} \cdots F_{r}=G_{1} \cdots G_{t}
$$

where $F_{1}, \ldots, F_{r}, G_{1}, \ldots, G_{t}$ are primitive functions. Without loss of generality, we may suppose that no $G_{i}$ is an $F_{1}$. But then,

$$
a_{p}\left(F_{1}\right)+\cdots+a_{p}\left(F_{r}\right)=a_{p}\left(G_{1}\right)+\cdots+a_{p}\left(G_{t}\right)
$$

so that

$$
\sum_{p \leq x} \frac{\overline{a_{p}\left(F_{1}\right)}\left(a_{p}\left(F_{1}\right)+\cdots+a_{p}\left(F_{r}\right)\right)}{p}=\sum_{p \leq x} \frac{\overline{a_{p}\left(F_{1}\right)}\left(a_{p}\left(G_{1}\right)+\cdots+a_{p}\left(G_{t}\right)\right)}{p} .
$$

As $x \rightarrow \infty$, the left-hand side tends to infinity, whereas the right-hand side is bounded, since no $G_{i}$ is an $F_{1}$. This contradiction proves:

Proposition 2.4 [CG]. Conjecture $B$ implies that every $F \in \mathscr{S}$ has a unique factorization into primitive functions.

In the next proposition, we describe $n_{F}$. 
Proposition 2.5. (a) If $F \in \mathscr{S}$ and $F=F_{1}^{e_{1}} \cdots F_{r}^{e_{r}}$ is a factorization into primitive functions, then Conjecture $B$ implies $n_{F}=e_{1}^{2}+\cdots+e_{r}^{2}$.

(b) Conjecture $B$ implies that $F$ is primitive if and only if $n_{F}=1$.

Proof. We have

$$
a_{p}(F)=\sum_{i=1}^{r} e_{i} a_{p}\left(F_{i}\right)
$$

so computing the asymptotic behavior of $\sum_{p \leq x}\left|a_{p}(F)\right|^{2} / p$ and using Conjecture $B$ yield the result.

\section{ARTIN'S CONJECTURE}

We begin by showing that Selberg's conjectures imply the holomorphy of nonabelian $L$-functions. In the notation of $\S 1$, let $\chi$ be the character of the representation $\rho$. We will write $L(s, \chi, K / k)$ for $L(s, \rho, K / k)$.

Theorem 3.1. Conjecture $B$ implies Artin's conjecture.

Proof. We adhere to the notation introduced in $\S 1$. Let $\widetilde{K}$ be the normal closure of $K$ over $Q$. Then, $\widetilde{K} / k$ is Galois, as well as $\widetilde{K} / Q$, and $\chi$ can be thought of as a character $\tilde{\chi}$ of $\operatorname{Gal}(\tilde{K} / k)$. By the property of Artin $L$-functions (see [A]),

$$
L(s, \tilde{\chi}, \tilde{K} / k)=L(s, \chi, K / k) .
$$

Moreover, if Ind $\tilde{\chi}$ denotes the induction of $\tilde{\chi}$ from $\operatorname{Gal}(\tilde{K} / k)$ to $\operatorname{Gal}(\tilde{K} / Q)$, then

$$
L(s, \tilde{\chi}, \tilde{K} / k)=L(s, \text { Ind } \tilde{\chi}, \tilde{K} / Q),
$$

by the invariance of Artin $L$-functions under induction. Hence, we can write

$$
L(s, \chi, K / k)=\prod_{\varphi} L(s, \varphi, \widetilde{K} / Q)^{m(\varphi)}
$$

where the product is over irreducible characters $\varphi$ of $\operatorname{Gal}(\widetilde{K} / Q)$ and $m(\varphi)$ are positive integers. To prove Artin's conjecture, it suffices to show that $L(s, \varphi, \widetilde{K} / Q)$ is entire for each irreducible character $\varphi$ of $\mathrm{Gal}(\widetilde{K} / Q)$. By Brauer's induction theorem and the Artin reciprocity law, we can write

$$
L(s, \varphi, \widetilde{K} / Q)=\frac{L\left(s, \chi_{1}\right)}{L\left(s, \chi_{2}\right)}
$$

where $\chi_{1}$ and $\chi_{2}$ are characters of $\operatorname{Gal}(\tilde{K} / Q)$ and $L\left(s, \chi_{1}\right), L\left(s, \chi_{2}\right)$ are entire functions, being products of Hecke $L$-functions. Thus, they belong to $\mathscr{S}$ and, hence, by Proposition 2.4 have a unique factorization into primitive functions. We can therefore write

$$
L(s, \varphi)=\prod_{i=1}^{m} F_{i}(s)^{e_{i}}, \quad e_{i} \in Z .
$$

By comparing the $p$ th Dirichlet coefficient of both sides, we get

$$
\varphi(p)=\sum_{i=1}^{m} e_{i} a_{p}\left(F_{i}\right)
$$


from which we obtain

$$
\sum_{p \leq x} \frac{|\varphi(p)|^{2}}{p}=\sum_{p \leq x} \frac{1}{p}\left|\sum_{i=1}^{m} e_{i} a_{p}\left(F_{i}\right)\right|^{2}
$$

Conjecture $\mathrm{B}$ gives the asymptotic behaviour of the right-hand side:

$$
\sum_{p \leq x} \frac{|\varphi(p)|^{2}}{p}=\left(\sum_{i=1}^{m} e_{i}^{2}\right) \log \log x+O(1) .
$$

Decompose the sum on the left-hand side according to the conjugacy class $C$ of $\operatorname{Gal}(\tilde{K} / Q)$ to which the Frobenius automorphism $\sigma_{p}$ belongs:

$$
\sum_{p \leq x} \frac{|\varphi(p)|^{2}}{p}=\sum_{C}\left|\varphi\left(g_{C}\right)\right|^{2} \sum_{\substack{p \leq x \\ \sigma_{p} \in C}} \frac{1}{p}
$$

where $g_{C}$ is any element of $C$. By the Chebotarev density theorem

$$
\sum_{\substack{p \leq x \\ \sigma_{p} \in C}} \frac{1}{p}=\frac{|C|}{|G|} \log \log x+O(1)
$$

Hence,

$$
\sum_{p \leq x} \frac{|\varphi(p)|^{2}}{p}=\sum_{C} \frac{|C|}{|G|}\left|\varphi\left(g_{C}\right)\right|^{2} \log \log x+O(1) .
$$

But $\varphi$ is irreducible, so

$$
\sum_{C} \frac{|C|}{|G|}\left|\varphi\left(g_{C}\right)\right|^{2}=(\varphi, \varphi)=1 .
$$

Therefore, the left-hand side is

$$
\log \log x+O(1)
$$

as $x \rightarrow \infty$. We deduce that $\sum_{i=1}^{m} e_{i}^{2}=1$, from which follows $m=1$ and $e_{1}= \pm 1$. Thus, $L(s, \varphi)=F(s)$ or $1 / F(s)$, where $F(s)$ is primitive and analytic everywhere except possibly at $s=1$. However, $L(s, \varphi)$ has trivial zeros, and so the latter possibility cannot arise. We conclude that $L(s, \varphi)=$ $F(s)$ is primitive and entire.

Corollary 3.2. Let $K / Q$ be Galois, and let $\chi$ be an irreducible character of $\operatorname{Gal}(K / Q)$. Conjecture $B$ implies that $L(s, \chi)$ is primitive.

Proof. This is evident from the last line in the proof of the previous lemma. Or we can derive it as follows. By the previous theorem, $F=L(s, \chi) \in \mathscr{S}$; and by the Chebotarev density theorem, $n_{F}=1$. The result now follows from Proposition 2.5(b).

Of course, Dedekind's conjecture that the zeta function of a number field is always divisible by the Riemann zeta function follows from Artin's conjecture. However, it is rather interesting to note that the unique factorization conjecture is sufficient to deduce this. Indeed, if $K$ is a number field, $\widetilde{K}$ its Galois closure, and $\zeta_{K}(s)$ is the Dedekind zeta function of $K$, then $\zeta_{\widetilde{K}}(s) / \zeta_{K}(s)=F(s)$ is entire by the Aramata-Brauer theorem. By the same theorem, $\zeta_{\widetilde{K}}(s) / \zeta(s)=$ 
$G(s)$ is also entire. Since $\zeta(s)$ is primitive, it appears as a primitive factor in $\zeta_{\widetilde{K}}(s)=\zeta(s) G(s)$. Since $\zeta_{\widetilde{K}}(s)=\zeta_{K}(s) F(s)$ and $F$ is entire, $\zeta(s)$ must appear in the unique factorization of $\zeta_{K}(s)$. This is Dedekind's conjecture.

\section{LANGLANDS RECIPROCITY CONJECTURE}

We begin by giving a very brief description of $L$-functions attached to automorphic representations of $G L_{n}$. Such a description is bound to be incomplete, so we refer the reader to [Co] for details. Let $n \geq 1, G=G L_{n}$, and $A$ be any commutative ring with identity. We denote by $G(A)$ the group of $n \times n$ matrices over $A$ whose determinant is a unit in $A$. Denote by $M_{r}$ a generic $r \times r$ matrix and by $I_{r}$ the $r \times r$ identity matrix. The standard parabolic subgroups of $G L_{n}$ are in one-to-one correspondence with the partitions of $n=n_{1}+\cdots+n_{r}$. The standard parabolic subgroup corresponding to a partition $n=n_{1}+\cdots+n_{r}$ consists of matrices of the form

$$
\left(\begin{array}{ccc}
M_{n_{1}} & * & * \\
& \ddots & * \\
& & M_{n_{r}}
\end{array}\right),
$$

and any parabolic subgroup is a $G L_{n}$ conjugate of a standard parabolic subgroup. Any parabolic subgroup $P$ has a decomposition (called the Levi decomposition) of the form $P=M N$ where $N$ is the unipotent radical of $P . M$ is called the Levi component of $P$. In the case of the standard parabolic, $M$ and $N$ can be described as consisting of matrices of the form

$$
\left(\begin{array}{ccc}
M_{n_{1}} & & \\
& \ddots & \\
& & M_{n_{r}}
\end{array}\right) \quad\left(\begin{array}{ccc}
I_{n_{1}} & * & * \\
& \ddots & * \\
& & I_{n_{r}}
\end{array}\right)
$$

respectively. We will write $N_{P}$ to denote the unipotent radical in the Levi decomposition of a parabolic subgroup $P$.

If $k$ is an algebraic number field, then the adele ring of $k$, denoted $A_{k}$, is a commutative ring with identity. It is defined in the following way. For each place $v$, let $k_{v}$ be the completion of $k$ at $v$. As a set $A_{k}$ consists of all infinite tuples $\left(x_{v}\right)$ where $v$ ranges over all places of $k$ with $x_{v}$ in $k_{v}$ and $x_{v}$ in the ring of $v$-adic integers, $O_{v}$ for all but finitely many places. One defines addition and multiplication componentwise which makes it into a commutative ring. We impose the adelic topology by declaring for each finite set $S$ of places containing the archimedean places,

$$
\prod_{v \in S} k_{v} \times \prod_{v \notin S} O_{v}
$$

with the product topology, as a basic neighborhood of the identity. This makes $A_{k}$ into a locally compact topological ring. One can think of $k$ as embedded in the ring $A_{k}$ via the map

$$
x \mapsto(x, x, \ldots) .
$$

The adelic topology on $G\left(A_{k}\right)$ is similarly defined. For each finite set $S$ as above, we declare

$$
\prod_{v \in S} G\left(k_{v}\right) \times \prod_{v \notin S} G\left(O_{v}\right)
$$


with the product topology, to be a basic neighborhood of the identity. The adelic topology on $G\left(A_{k}\right)$ makes it into a locally compact group in which $G(k)$, embedded diagonally, is a discrete subgroup of $G\left(A_{k}\right)$. The coset space, $G(k) \backslash G\left(A_{k}\right)$, with quotient topology does not have finite volume with respect to any $G\left(A_{k}\right)$ invariant measure. To rectify this, define

$$
Z=\left\{\left(\begin{array}{ccc}
z & & \\
& \ddots & \\
& & z
\end{array}\right): z \in A_{k}^{\times}\right\} .
$$

Then, the quotient $Z G(k) \backslash G\left(A_{k}\right)$ has finite volume with respect to any $G\left(A_{k}\right)$ invariant measure.

A character of $k^{\times} \backslash G L_{1}\left(A_{k}\right)$ is called a Grossencharacter. Now fix a Grossencharacter $\omega$ of $k$. We can consider the Hilbert space

$$
L^{2}\left(G(k) \backslash G\left(A_{k}\right), \omega\right)
$$

of measurable functions $\varphi$ on $G(k) \backslash G\left(A_{k}\right)$ satisfying

(i) $\varphi(z g)=\omega(z) \varphi(g), z \in Z, g \in G(k) \backslash G\left(A_{k}\right)$;

(ii) $\int_{Z G(k) \backslash G\left(A_{k}\right)}|\varphi(g)|^{2} d g<\infty$.

The subspace of cusp forms $L_{0}^{2}\left(G(k) \backslash G\left(A_{k}\right), \omega\right)$ is defined by the extra condition

(iii) for all parabolic subgroups $P$ of $G\left(A_{k}\right)$,

$$
\int_{N_{P}(k) \backslash N_{P}\left(A_{k}\right)} \varphi(n g) d n=0
$$

for every $g \in G\left(A_{k}\right)$. Thus,

Let $R$ be the right regular representation of $G\left(A_{k}\right)$ on $L^{2}\left(G(k) \backslash G\left(A_{k}\right), \omega\right)$.

$$
(R(g) \varphi)(x)=\varphi(x g)
$$

for $\varphi \in L^{2}\left(G(k) \backslash G\left(A_{k}, \omega\right)\right.$ and $x, g \in G\left(A_{k}\right)$. This is a unitary representation of $G\left(A_{k}\right)$. An automorphic representation is a subquotient of the right regular representation of $G\left(A_{k}\right)$ on $L^{2}\left(G(k) \backslash G\left(A_{k}\right), \omega\right)$. A cuspidal automorphic representation is a subrepresentation of the right regular representation of $G\left(A_{k}\right)$ on $L_{0}^{2}\left(G(k) \backslash G\left(A_{k}\right), \omega\right)$.

A representation of $G\left(A_{k}\right)$ is called admissible if its restriction to the maximal compact subgroup

$$
K=\prod_{v \text { complex }} U(n, C) \times \prod_{v \text { real }} O(n, R) \times \prod_{v \text { finite }} G L_{n}\left(O_{v}\right)
$$

contains each irreducible representation of $K$ with only finite multiplicity.

To understand the structure of these representations, we need the notion of a restricted tensor product of representations originally introduced in [JL]. Let $\left\{W_{v} \mid v \in V\right\}$ be a family of vector spaces indexed by the set $V$. Let $V_{0}$ be a finite subset of $V$. For each $v \in V \backslash V_{0}$, let $x_{v}$ be a nonzero vector in $W_{v}$. For each finite subset $S$ of $V$ containing $V_{0}$, let $W_{s}=\bigotimes_{v \in S} W_{v}$. If $S \subset S^{\prime}$, let $f_{S, S^{\prime}}: W_{S} \rightarrow W_{S^{\prime}}$ be defined by

$$
\bigotimes_{v \in S} w_{v} \mapsto \bigotimes_{v \in S} w_{v} \bigotimes_{v \in S^{\prime} \backslash S} x_{v}
$$


Then, the restricted tensor product of the $W_{v}$ with respect to the $x_{v}, W=$ $\bigotimes_{x_{v}} W_{v}$, is defined to be the inductive limit $W=$ ind $\lim _{S} W_{S}$. It is known [F, p. 181] that any irreducible, admissible representation of $G L_{n}\left(A_{k}\right)$ can be written as a restricted tensor product $\bigotimes_{v} \pi_{v}$ where $\pi_{v}$ is an irreducible representation of $G L_{n}\left(k_{v}\right)$. Moreover, the factors $\pi_{v}$ are unique up to equivalence.

For each finite $v$, one can construct certain representations of $G L_{n}\left(k_{v}\right)$ in the following way. One has the Borel subgroup

$$
B\left(k_{v}\right)=\left\{b=\left(\begin{array}{ccc}
b_{1} & * & * \\
& \ddots & * \\
& & b_{n}
\end{array}\right)\right\} \subseteq G L_{n}\left(k_{v}\right)
$$

if $G L_{n}\left(k_{v}\right)$. For any $n$-tuple, $z=\left(z_{1}, \ldots, z_{n}\right) \in C^{n}$, define $\chi_{z}$ on $B\left(k_{v}\right)$ by

$$
\chi_{z}(b)=\left|b_{1}\right|_{v}^{z_{1}} \cdots\left|b_{n}\right|_{v}^{z_{n}}
$$

where $|\cdot|_{v}$ denotes the $v$-adic metric. This gives a quasi-character on $B\left(k_{v}\right)$. Let $\tilde{\pi}_{v, z}$ be the representation of $G L_{n}\left(k_{v}\right)$ obtained by inducing $\chi_{z}$ from $B\left(k_{v}\right)$ to $G L_{n}\left(k_{v}\right)$. Recalling the definition of an induced representation, we see that this means the following: it is the space of locally constant functions $\varphi$ on $G L_{n}\left(k_{v}\right)$ such that for $b \in B\left(k_{v}\right), x \in G L_{n}\left(k_{v}\right)$,

$$
\varphi(b x)=\chi_{z}(b)\left(\prod_{i=1}^{n}\left|b_{i}\right|_{v}^{(n+1) / 2-i}\right) \varphi(x),
$$

and $\tilde{\pi}_{v, z}$ acts on $\varphi$ via

$$
\left(\tilde{\pi}_{v, z}(y) \varphi\right)(x)=\varphi(x y)
$$

We shall assume that

$$
\operatorname{Re}\left(z_{1}\right) \geq \operatorname{Re}\left(z_{2}\right) \geq \cdots \geq \operatorname{Re}\left(z_{n}\right) .
$$

Then, a special case of the Langlands classification [BW, Chapter 11, §2] shows that $\tilde{\pi}_{v, z}$ has a unique irreducible quotient $\pi_{v, z}$. The representations obtained in this way are called the unramified principal series. They are precisely the representations of $G L_{n}\left(k_{v}\right)$ whose restriction to $G L_{n}\left(O_{v}\right)$ contains the trivial representation. If $\pi_{v}$ is any representation of $G L_{n}\left(k_{v}\right)$ equivalent to $\pi_{v, z}$, we can define

$$
A_{v}=\operatorname{diag}\left(N v^{-z_{1}}, \ldots, N v^{-z_{n}}\right) \in G L_{n}(C)
$$

where $N v$ denotes the norm of $v$. One can show that $A_{v}$ depends only on the equivalence class of $\pi_{v}$.

Now let $\pi=\bigotimes_{v} \pi_{v}$ be an irreducible, admissible, automorphic representation of $G L_{n}\left(A_{k}\right)$. Since $\pi$ is admissible, there is a finite set $S$ of places (containing the infinite places) such that $\pi_{v}$ belongs to the unramified principal series for $v \notin S$. For such $v$, define

$$
L_{v}(s, \pi)=\operatorname{det}\left(1-A_{v} N v^{-s}\right)^{-1} .
$$

Set

$$
L_{S}(s, \pi)=\prod_{v \notin S} L_{v}(s, \pi)
$$


Using the theory of Eisenstein series, Langlands [L3] established the meromorphic continuation of $L_{S}(s, \pi)$. It is possible to define $L_{v}\left(s, \pi_{v}\right)$ for $v \in S$ such that the complete $L$-function

$$
L(s, \pi)=\prod_{v} L_{v}\left(s, \pi_{v}\right)
$$

has a meromorphic continuation and functional equation. Moreover, if $\pi$ is cuspidal, then $L(s, \pi)$ extends to an entire function unless $n=1$ and $\pi$ is of the form $|\cdot|^{t}$ for some $t \in C$. This was proved by Godement and Jacquet [GJ]. The Ramanujan conjecture asserts that the eigenvalues of $A_{v}$ are of absolute value 1 for cuspidal automorphic representations. This is still one of the main open problems in the theory of automorphic representations. If the Ramanujan conjecture is true, then these $L$-functions belong to the Selberg class.

For $n=1$ this is the classical theory of Hecke's $L$-series attached to Grossencharacters, and Tate's thesis offers the important perspective of an adelic reformulation of Hecke's work. For $n=2$ and $K=Q$, the analytic continuation and functional equation of the corresponding $L$-function are due to Hecke and Maass. The generalization to global fields and the adelic formulation is the work of Jacquet and Langlands [JL]. The general case for $G L_{n}$ is due to Godement and Jacquet [GJ]. For $n=3$, a simple proof can be found in the work of Hoffstein and Murty [HM]. A gentle introduction to the Langlands program can be found in [M1].

In this adelic framework, Langlands conjectures that every Artin $L$-series $L(s, \rho)$ is an $L(s, \pi)$ for some automorphic representation $\pi$ on $G L_{n}\left(A_{k}\right)$ where $n=\operatorname{dim} \rho$. Thus, to each $\rho$ there should be an automorphic representation $\pi(\rho)$. In case $\operatorname{deg} \rho=1$, this is Artin's reciprocity law, since Hecke's Grossencharacters are automorphic representations of $G L_{1}\left(A_{k}\right)$.

Let $K / k$ be a Galois extension, and let $G=\operatorname{Gal}(K / k)$. Let $H$ be a subgroup of $G$ and $K^{H}$ its fixed field. If $\psi$ is a representation of $H$, then $L\left(s, \psi, K / K^{H}\right)$ is the Artin $L$-series belonging to the extension $K / K^{H}$. We have already noted that Artin $L$-series are invariant under induction:

$$
L\left(s, \operatorname{Ind}_{H}^{G} \psi, K / k\right)=L\left(s, \psi, K / K^{H}\right) .
$$

If the Langlands reciprocity conjecture is true, this last property should imply a corresponding property for $L$-series attached to automorphic representations. This leads to the concept of the base change $L$-function which we now define.

To this end, let us first describe how the map $\rho \mapsto \pi(\rho)$ behaves under restriction to a subgroup. If we denote by $\mathscr{A}\left(G L_{n}\left(A_{k}\right)\right)$ the space of automorphic representations of $G L_{n}\left(A_{k}\right)$, then by the reciprocity conjecture, we should have

$$
\left.\rho\right|_{H} \mapsto \pi\left(\left.\rho\right|_{H}\right) \in \mathscr{A}\left(G L_{n}\left(A_{K^{H}}\right)\right) .
$$

What is $L\left(s,\left.\rho\right|_{H}, K / K^{H}\right)$ ? Since

$$
\operatorname{Ind}_{H}^{G}\left(\left.\rho\right|_{H} \otimes \psi\right)=\rho \otimes \operatorname{Ind}_{H}^{G} \psi,
$$

we find

$$
L\left(s, \operatorname{Ind}_{H}^{G}\left(\left.\rho\right|_{H} \otimes \psi\right), K / k\right)=L\left(s, \rho \otimes \operatorname{Ind}_{H}^{G} \psi, K / k\right) .
$$

But the former $L$-function is $L\left(s,\left.\rho\right|_{H} \otimes \psi, K / K^{H}\right)$ by the invariance of Artin $L$-series. Therefore,

$$
L\left(s,\left.\rho\right|_{H}, K / K^{H}\right)=L\left(s, \rho \otimes \operatorname{Ind}_{H}^{G} 1, K / k\right) .
$$


But $\operatorname{Ind}_{H}^{G} 1=\operatorname{reg}_{G / H}$ is the permutation representation on the cosets of $H$. This suggests that we make the following definition. Let $\pi \in \mathscr{A}\left(G L_{n}\left(A_{k}\right)\right)$. Define, for unramified $v$,

$$
L_{v}(s, B(\pi))=\operatorname{det}\left(1-A_{v} \otimes \operatorname{deg}_{G / H}\left(\sigma_{v}\right) N v^{-s}\right)^{-1},
$$

where $\sigma_{v}$ is the Artin symbol of $v$. For ramified $v$, see [L2] or [AC] for the definition of $L_{v}(s, B(\pi))$. Set

$$
L(s, B(\pi))=\prod_{v} L_{v}(s, B(\pi)) .
$$

$B(\pi)$ should correspond to an element of $\mathscr{A}\left(G L_{n}\left(A_{M}\right)\right)$ where $M=K^{H}$. The problem of base change is to determine when this map exists.

For $n=2$ and $M / k$ cyclic, this is a theorem of Langlands [L2]. For arbitrary $n, M / k$ cyclic, it is the main theorem of Arthur and Clozel [AC].

The reciprocity conjecture forces another conjectural property for the automorphic $L$-functions. Suppose that $\psi$ is a representation of $H$. Corresponding to $\psi$ there should be a $\pi \in \mathscr{A}\left(G L_{n}\left(A_{M}\right)\right)$ where $n=\operatorname{dim} \psi$. But the invariance of Artin $L$-series under induction implies that there should be an $I(\pi) \in \mathscr{A}\left(G L_{n r}\left(A_{k}\right)\right) \quad(r=[G: H])$ so that

$$
L(s, I(\pi))=L\left(s, \operatorname{Ind}_{H}^{G} \psi, K / k\right) .
$$

This map $\pi \mapsto I(\pi)$, called the automorphic induction map, is conjectured to exist. Again, Arthur and Clozel [AC] showed this exists when $M / k$ is cyclic and arbitrary $n$. Thus, if $M / k$ is contained in a solvable extension of $k$, the base change maps and automorphic induction maps exist. We can summarize this discussion in the following.

Lemma 4.1 [AC]. Let $E / F$ be a cyclic extension of prime degree $p$ of algebraic number fields, and let $\pi$ be an automorphic representation of $G L_{n}\left(A_{F}\right)$. Then there is the base change lift $B(\pi)$ of $\pi$ such that $B(\pi)$ is an automorphic representation of $G L_{n}\left(A_{E}\right)$. If $\sigma$ is an automorphic representation of $G L_{n}\left(A_{E}\right)$, then the automorphic induction $I(\sigma)$ exists.

We now have the requisite background to prove:

Lemma 4.2. Assume Conjecture $B$. If $\pi$ is an irreducible cuspidal automorphic representation of $G L_{n}\left(A_{Q}\right)$ that satisfies the Ramanujan conjecture, then $L(s, \pi)$ is primitive.

Proof. Let $\pi_{1}$ and $\pi_{2}$ be two irreducible cuspidal automorphic representations of $G L_{n}\left(A_{Q}\right)$. We recall the work of Jacquet and Shalika [JS1, JS2] and Shahidi [Sh]. Let $S$ be a finite set of primes such that $\pi_{1}, \pi_{2}$ are unramified outside $S$. We form the $L$-function

$$
L_{S}\left(s, \pi_{1} \otimes \pi_{2}\right)=\prod_{v \notin S} \operatorname{det}\left(1-A_{v, 1} \otimes A_{v, 2} N v^{-s}\right)^{-1},
$$

where $A_{v, 1}$ and $A_{v, 2}$ are the diagonal matrices associated to $\pi_{1}$ and $\pi_{2}$ respectively, as indicated above. Then, we will use the following properties, derived in [JS1, JS2]:

(a) The Euler product $L_{S}$ is absolutely convergent for $\operatorname{Re} s>1$.

(b) Let $X$ be the set of $s$ on the line $\operatorname{Re} s=1$ such that $\pi_{1} \otimes|\cdot|^{s-1}$ is equivalent to $\tilde{\pi}_{2}$, the contragredient of $\pi_{2}$. (Thus, $X$ contains at most one 
point.) Then the function $L_{S}$ extends continuously to the line $\operatorname{Re} s=1$ with $X$ removed. Moreover, it does not vanish there.

(c) If $s_{0} \in X$, the limit

$$
\lim _{\substack{s \rightarrow s_{0} \\ \operatorname{Re} s \geq 1}}\left(s-s_{0}\right) L_{S}\left(s, \pi_{1} \otimes \pi_{2}\right)
$$

exists and is finite and nonzero.

These facts are proved in [JS1, Theorem 5.3, JS2, Proposition 3.6], and the nonvanishing results are proved by Shahidi [Sh]. When $\pi_{1}$ and $\tilde{\pi}_{2}$ are distinct, this means that $L_{S}\left(s, \pi_{1} \otimes \pi_{2}\right)$ is analytic and nonvanishing for $\operatorname{Re} s=1$, whereas $L_{S}\left(s, \pi_{1} \otimes \tilde{\pi}_{1}\right)$ has a simple pole at $s=1$ and is nonvanishing for $\operatorname{Re} s=1$. In analytic terms, this implies

$$
\sum_{p \leq x} \frac{\left|a_{p}\left(\pi_{i}\right)\right|^{2}}{p}=\log \log x+O(1)
$$

and

$$
\sum_{p \leq x} \frac{a_{p}\left(\pi_{1}\right) \overline{a_{p}\left(\pi_{2}\right)}}{p}=O(1) .
$$

By Proposition 2.5(b), we deduce the desired result.

We are now ready to prove the main theorem of this section.

Theorem 4.3. Assume Conjecture B. Let $K$ be a Galois extension of $Q$ with solvable group $G$, and let $\chi$ be an irreducible character of $G$ of degree $n$. Then there is an irreducible cuspidal automorphic representation $\pi$ of $G L_{n}\left(A_{Q}\right)$ such that $L(s, \chi)=L(s, \pi)$.

Proof. The Dedekind zeta function of $K$ is a Hecke $L$-function. In fact, it is an automorphic $L$-function of $G L_{1}\left(A_{K}\right)$. Since $K$ is solvable over $Q$, there is a chain of cyclic extensions,

$$
Q=K_{0} \subset K_{1} \cdots \subset K_{m-1} \subset K_{m}=K
$$

so that $\left[K_{i}: K_{i-1}\right]$ has prime degree. By Lemma 4.1 and automorphic induction, we can identify $\zeta_{K}(s)$ as an automorphic $L$-function, first of $K_{m-1}$ and then successively of $K_{i}$ until we deduce that it is an automorphic $L$-function of $Q$. Thus, as in $[\mathrm{AC}]$, we have the factorization

$$
\zeta_{K}(s)=\prod_{i=1}^{r} L\left(s, \pi_{i}\right)^{e_{i}}
$$

where $\pi_{i}$ are irreducible cuspidal automorphic representations of appropriate degree over $Q$ and $e_{i}$ are positive integers. Since the eigenvalues of the $p$ Euler factors of the left-hand side are of absolute value 1, so are the eigenvalues of the $p$-Euler factors on the right-hand side. Thus, each $L\left(s, \pi_{i}\right)$ satisfies the Ramanujan conjecture. By Lemma 4.2, each factor is primitive. Thus, the above is the unique factorization of $\zeta_{K}(s)$ into primitive factors. On the other hand, we have the classical factorization

$$
\zeta_{K}(s)=\prod_{\chi} L(s, \chi)^{\chi(1)}
$$


where the product is over the irreducible characters of $G$. By Theorem 3.1 and Corollary 3.2, each $L(s, \chi)$ is in $\mathscr{S}$ and, moreover, primitive. Thus, each $L(s, \chi)$ must be an $L(s, \pi)$ by uniqueness of factorization. This is the reciprocity law.

Remark 4.4. If $K$ were an arbitrary Galois extension of $Q$ and we knew that $\zeta_{K}(s)$ was automorphic, the reciprocity conjecture would follow in the same way on the assumption of Conjecture B.

\section{CONCLUding Remarks}

The Selberg conjectures refer to the analytic behavior of Dirichlet series at the edge of the critical strip. There are other conjectures relating special values of Dirichlet series inside the critical strip, namely, the Deligne conjectures and the Birch-Swinnerton-Dyer conjectures to cite specific instances. A consequence of Conjecture $B$ is that if $F$ is any primitive function which is not the Riemann zeta function, then

$$
\sum_{p \leq x} \frac{a_{p}(F)}{p^{1+i t}}=O(1)
$$

In particular, no primitive function should vanish on $\sigma=1$.

Many of our interesting consequences, notably the Artin conjecture and the Langlands reciprocity conjecture, utilized the unique factorization conjecture. Perhaps this can be attacked by other means. Indeed, given $r$ distinct primitive functions $F_{1}, \ldots, F_{r}$, one would expect the existence of complex numbers $s_{1}, \ldots, s_{r}$ such that $F_{i}\left(s_{j}\right)=0$ if and only if $i=j$. If this were the case, then clearly, the unique factorization conjecture would be true.

The classification of primitive functions is a fundamental problem. From the work of Bochner and Vignéras, it follows that if $F$ has dimension 1 and all the $\alpha_{i}$ are rational numbers, then $d=1$ and $\alpha_{1}=1 / 2$. It then follows, essentially from the same works, that $F$ must either be the Riemann zeta function or a purely imaginary translate of a classical Dirichlet $L$-function attached to a nontrivial primitive character. It is shown in [M2] that if $\pi$ is an irreducible cuspidal automorphic representation of $G L_{2}\left(A_{Q}\right)$, then $L(s, \pi)$ is primitive if the Ramanujan conjecture is true. In particular, the $L$-function attached to a normalized holomorphic cuspidal Hecke eigenform is a primitive function which is in Selberg's class (by Deligne's theorem).

\section{ACKNOWLEDGMENT}

I would like to thank Professors Barry Mazur, J.-P. Serre, and Dipendra Prasad for their critical comments on an earlier version of this paper.

\section{REFERENCES}

[AC] J. Arthur and L. Clozel, Simple algebras, base change and the advanced theory of the trace formula, Ann. of Math. Stud., vol. 120, Princeton Univ. Press, Princeton NJ, 1990.

[A] E. Artin, Collected papers, Addison-Wesley, Reading, MA, 1965.

[B] S. Bochner, On Riemann's functional equation with multiple gamma factors, Ann. of Math. (2) 67 (1958), 29-41.

[BW] A. Borel and N. Wallach, Continuous cohomology, discrete subgroups and representations of reductive groups, Ann. of Math. Stud., vol. 94, Princeton Univ. Press, Princeton, NJ, 1980. 
[CG] B. Conrey and A. Ghosh, Selberg class of Dirichlet series: Small degrees, Duke Math. J. 72 (1993), 673-693.

[Co] - Automorphic forms, representations and L-functions, Proc. Sympos. Pure Math., vol. 33, Amer. Math. Soc., Providence, RI, 1979.

[F] D. Flath, Decomposition of representations into tensor products, Automorphic Forms, Representations and $L$-functions, vol. 33, Amer. Math. Soc., Providence, RI, pp. 179-184.

[GJ] R. Godement and H. Jacquet, Zeta functions of simple algebras, Lecture Notes in Math., vol. 260, Springer-Verlag, Berlin and New York, 1972.

[HM] J. Hoffstein and R. Murty, L-series of automorphic forms on $G L_{3}(R)$, Number Theory (J.-M. de Koninck and C. Levesque, eds.), Walter de Gruyter, Berlin and New York, 1989.

[JL] H. Jacquet and R. Langlands, Automorphic forms on $G L(2)$, Lecture Notes in Math., vol. 114, Springer-Verlag, Berlin and New York, 1970.

[JS1] H. Jacquet and J. A. Shalika, On Euler products and the classification of automorphic representations. I, Amer. J. Math 103 (1981), 499-558.

[JS2] - On Euler products and the classification of automorphic representations. II, Amer. J. Math. 103 (1981), 777-815.

[L1] R. Langlands, Problems in the theory of automorphic forms, Lecture Notes in Math., vol. 170, Springer-Verlag, Berlin and New York, 1970.

[L2] Base change for $G L(2)$, Ann. of Math. Stud., vol. 96, Princeton Univ. Press, Princeton, NJ, 1980.

[L3] - On the functional equations satisfied by Eisenstein series, Lecture Notes in Math., vol. 544, Springer-Verlag, New York, 1976.

[M1] M. Ram Murty, A motivated introduction to the Langlands program, Advances in Number Theory (F. Gouvea and N. Yui, eds.), Clarendon Press, Oxford, 1993, pp. 37-66.

[M2] - Selberg conjectures and Artin L-functions. II (to appear).

[S] A. Selberg, Old and new conjectures and results about a class of Dirichlet series, Collected Papers, Volume II, Springer-Verlag, 1991, pp. 47-63.

[Sh] F. Shahidi, On non-vanishing of L-functions, Bull. Amer. Math. Soc. (N.S.) 2 (1980), 462464.

[V] M. F. Vignéras, Facteurs gamma et équations fonctionelles, Lecture Notes in Math., vol. 627, Springer-Verlag, Berlin and New York, 1976.

Department of Mathematics, McGill University, Montreal, H3A 2K6 Canada

E-mail address: murty@math.mcgill.ca 